\title{
On the Study of Four-Parallelogram Filter Banks
}

\author{
Yuan-Pei Lin, Student Member, IEEE, and P. P. Vaidyanathan, Fellow, IEEE
}

\begin{abstract}
The most commonly used 2-D filter banks are separable filter banks, which can be obtained by cascading two 1-D filter banks in the form of a tree. The supports of the analysis and synthesis filters in the separable systems are unions of four rectangles. The natural nonseparable generalization of such supports are those that are unions of four parallelograms. In this paper, we study four parallelogram filter banks, which is the class of 2-D filter banks in which the supports of the analysis and synthesis filters consist of four parallelograms. For a given a decimation matrix, there could be more than one possible configuration (the collection of passbands of the analysis filters). Various types of configuration will be constructed for four-parallelogram filter banks. Conditions on the configurations will be derived such that good design of analysis and synthesis filters are possible. We will see that there is only one category of these filter banks. The configurations of four-parallelogram filter banks in this category can always be achieved by designing filter banks of low design cost.
\end{abstract}

\section{INTRODUCTION}

$\mathbf{I}^{\mathbf{N}}$ $\mathrm{N}$ a companion paper [1], we studied 2-D twoparallelogram filter banks. This is the class of systems in which the supports (passbands) of the analysis filters consist of two parallelograms, each a shifted version of a parallelogram prototype. Fig. 1(a) and (b) show, respectively, a parallelogram prototype and the support (passband) of a typical analysis filter in a two-parallelogram filter bank. The analysis filters have real coefficients; the supports are symmetric with respect to the origin. Consider the 2-D filter bank with decimation matrix $\boldsymbol{M}$ in Fig. 2. It is explained in [1] that for successful design of the analysis and synthesis filters, it is necessary that the configuration of the filter bank be $\mathcal{A} \mathcal{F} \mathcal{S}(\boldsymbol{M})$ and permissible. More specific description of these two properties are given below.

A filter $H(\omega)$ is called aliasfree supported with respect to $\boldsymbol{M}(\mathcal{A} \mathcal{F} \mathcal{S}(\boldsymbol{M}))$ if the support of $H(\omega)$ does not overlap under modulo $2 \pi M^{-T}$. When $H(\boldsymbol{\omega})$ is an ideal filter, the output of $H(\omega)$ would allow aliasfree $\boldsymbol{M}$-fold decimation. When all the analysis filters are $\mathcal{A} \mathcal{F} \mathcal{S}(\boldsymbol{M})$, the configuration is referred to as $\mathcal{A} \mathcal{F} \mathcal{S}(\boldsymbol{M})$. If a filter bank has a $\mathcal{A} \mathcal{F} \mathcal{S}(\boldsymbol{M})$ configuration, then, in the ideal case, no aliasing is created in the subbands, and the filter bank has perfect reconstruction. In practice, the nonideal rolloff of the filter causes aliasing in the subband. When the analysis filters are decimated and then expanded by $\boldsymbol{M}$, each has $|\boldsymbol{M}|-1$ images, where the notation $|\boldsymbol{M}|$ denotes the absolute value of the determinant of

Manuscript received November 26, 1995; revised April. 14, 1996. This work was supported, in part, by NSF grant MIP 92-15785, Tektronix, Inc., and Rockwell International. The associate editor coordinating the review of this paper and approving it for publication was Dr. Bruce Suter.

The authors are with the Department of Electrical Engineering, California Institute of Technology, Pasadena, CA 91125 USA.

Publisher Item Identifier S 1053-587X(96)08237-2.

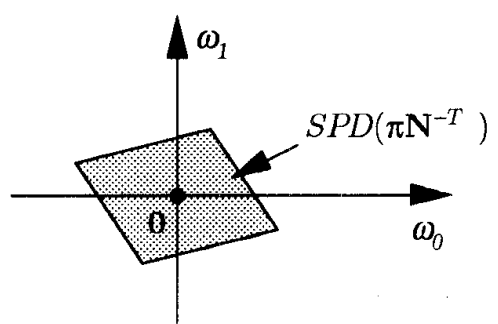

(a)

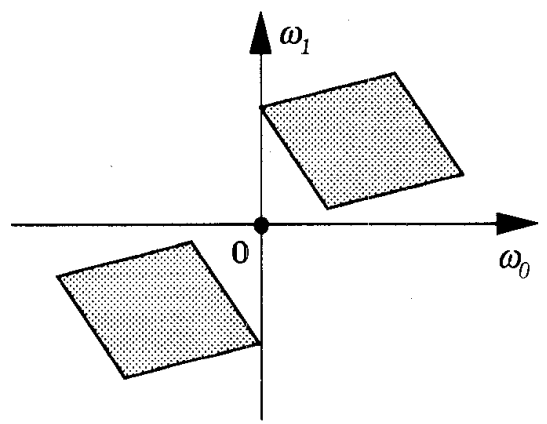

(b)

Fig. 1. Two-parallelogram filter bank. (a) Parallelogram prototype and (b) typical support of an analysis filter.

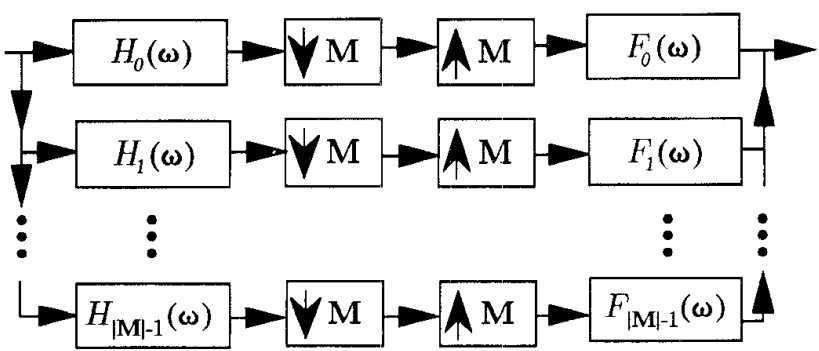

Fig. 2. $|\boldsymbol{M}|$-channel maximally decimated filter bank, where $|\boldsymbol{M}|$ denotes the absolute value of the determinant of $M$.

$M$. All the images are attenuated to the stopband level of the synthesis filters except those images that are adjacent to the passband of the synthesis filters. These adjacent images result in different types of major aliasing, depending on the type of adjacency involved. For example, in Fig. 3(a), one image of the analysis filter $H_{k}(\boldsymbol{\omega})$ is edge adjacent to the synthesis filter $F_{k}(\omega)$ and results in edge aliasing, and similarly, the image in Fig. 3(b) results in vertex aliasing. A perfect reconstruction filter bank enjoys complete alias cancelation; therefore, major aliasing of one subband is canceled largely by major aliasing of other subbands. However, when the configurations are not constructed properly, it is possible that some edge aliasing 


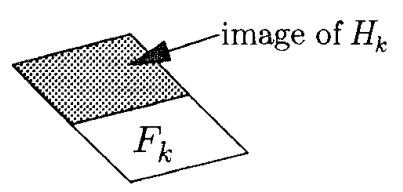

(a)

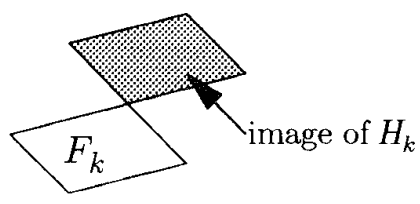

(b)

Fig. 3. (a) Image of the kith analysis filter is edge ajacent to the $k$ th synthesis filter. (b) Image of the $k$ th analysis filter is vertex ajacent to the $k$ th synthesis filter.

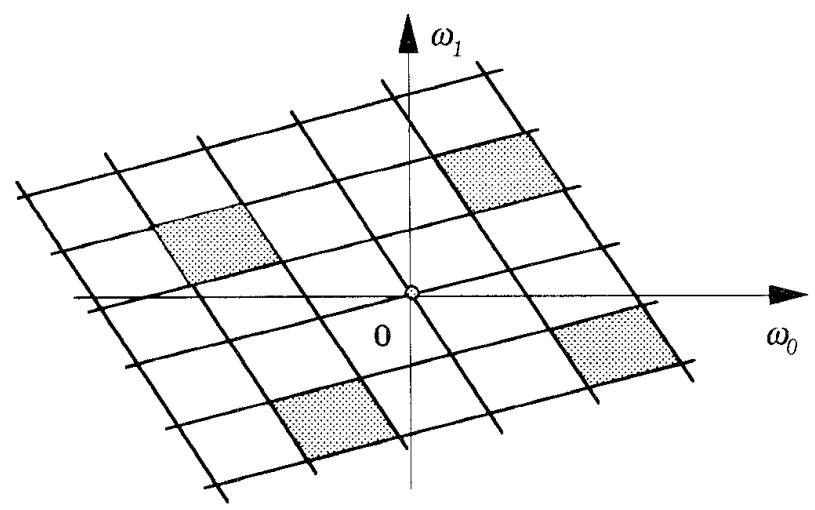

Fig. 4. Typical support of an analysis filter in a four-parallelogram filter bank.

cannot be canceled if the analysis and synthesis filters have good frequency selectivity. Such configurations are called edge nonpermissible. Similarly, if some vertex aliasing in a configuration are uncancelable when the filters have good frequency selectivity, the configuration is called vertex nonpermissible. For the individual filters to have good frequency selectivity, it is necessary that the configuration have permissibility [2], [3], which includes edge and vertex permissibility [1]. In this case, the importance of edge permissibility is much greater than vertex permissibility. Notice that permissibility is determined jointly by all the analysis filters. To have a permissible configuration, the support of one analysis filter will affect the choice of other filters.

Although the two-parallelogram filter banks cannot possess both edge and vertex permissibility in general, the twoparallelogram filter banks can have edge permissibility. Edge permissible two-parallelogram CMFB's are constructed and designed in [1] and [4]. However, lack of permissibility lead to limitation in the stopband attenuation of the individual filters in the two-parallelogram cosine modulated filter banks.

\section{Four-Parallelogram Filter Banks}

In this paper, we study four parallelogram filter banks [5], the class of filter banks in which the supports of the analysis filters consist of four parallelograms as shown in

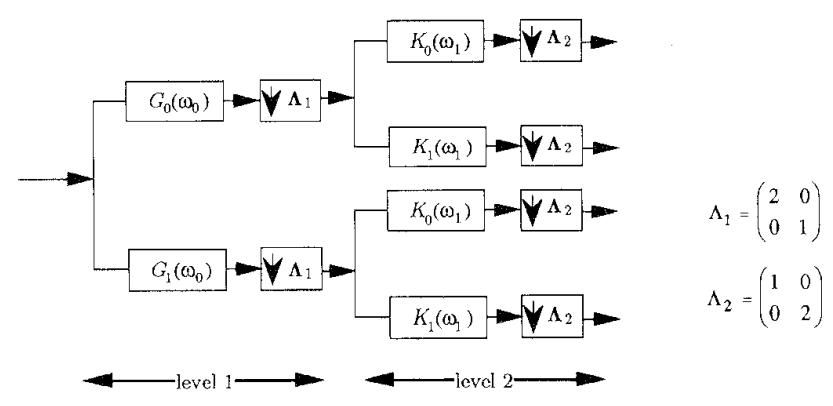

Fig. 5. Four-channel tree-structured filter bank obtained by cascading two 1-D two-channel filter banks.

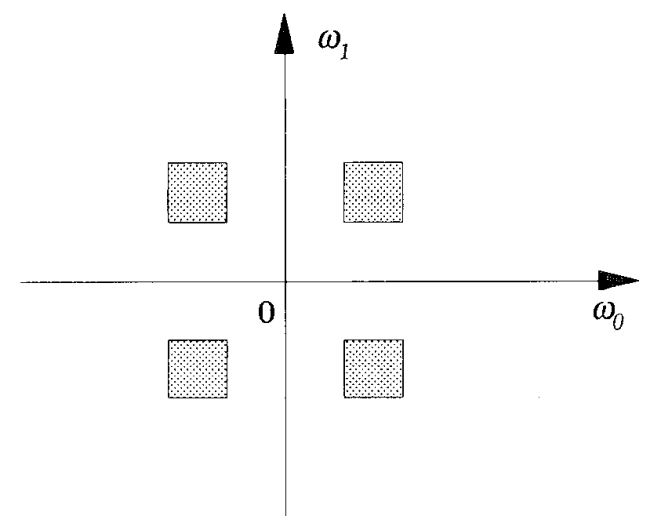

Fig. 6. Separable filter bank. Typical support of an analysis filter.

Fig. 4. The simplest way to design four-parallelogram filter banks is by using separable filter banks [6]. A separable 2D filter bank can be obtained by cascading two 1-D filter banks in a tree structure. For example, the tree-structured filter bank in Fig. 5 is obtained by using two 1-D two-channel filter banks. The resulting 2-D analysis and synthesis filters are products of two 1-D filters and are separable; the support of each analysis filter is the union of four rectangles (Fig. 6). The 2-D analysis and synthesis filters can have good frequency selectivity if the 1-D filters have good frequency selectivity. Therefore, the separable 2-D filter banks are both edge and vertex permissible. In this paper, we will pursue edge and vertex permissibility for four-parallelogram filter banks.

To obtain a support configuration for four-parallelogram filter bank, we first construct a parallelogram prototype. Then, we shift the parallelogram prototype properly and combine four shifted parallelograms to obtain the support of each analysis filter. The synthesis filters have the same supports as the corresponding analysis filters. As the support of each analysis filter consists of four shifted versions of the parallelogram prototype, the area of the parallelogram prototype is one fourth the area of the support of an analysis filter. In a $|M|$ channel filter bank, the area of an analysis filter support is usually the same as $\operatorname{SPD}\left(\pi \boldsymbol{M}^{-T}\right)$, where $S P D(\boldsymbol{V})$ of a $2 \times 2$ matrix $\boldsymbol{V}$ is the set of vectors of the form [7]

$$
\boldsymbol{V} \boldsymbol{x}, \boldsymbol{x} \in[-1,1)^{2} \text {. }
$$

Let the parallelogram prototype be $\operatorname{SPD}\left(\pi \boldsymbol{N}^{-T}\right)$, where $N=M L$ for some integer matrix $L$ with $|L|=4$. Due 


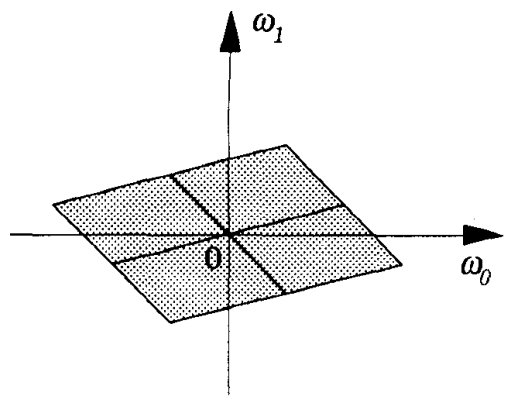

(a)

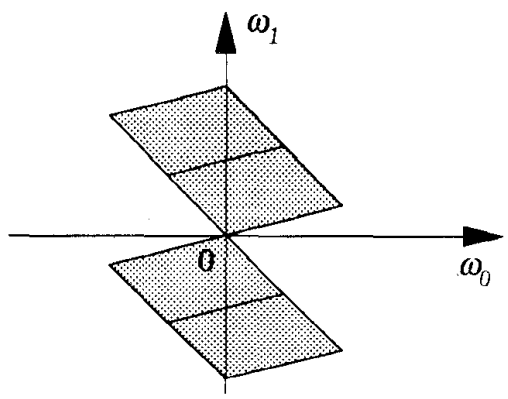

(b)

Fig. 7. Two of the possible supports for the zeroth analysis filter.

to the integer matrix constraint on $L$, the four-parallelogram filter banks considered in this paper are only a subclass of four-parallelogram filter banks. Since $|\boldsymbol{N}|=4|\boldsymbol{M}|$, the area of $S P D\left(\pi N^{-T}\right)$ is one fourth that of $S P D\left(\pi M^{-T}\right)$. For a given decimation matrix $M$, the choice of $L$ will determine the parallelogram prototype and indirectly affect the supports of the analysis filters. Then, we combine four shifted versions of the parallelogram prototype to obtain the supports of the analysis filters.

For four-parallelogram filter banks, there are a variety of possible configurations. For example, consider the lowpass analysis filter $H_{0}(\boldsymbol{\omega})$. Fig. 7(a) and (b) show two of the possible supports for $H_{0}(\omega)$. The support of $H_{0}(\omega)$ is different when the four parallelograms in the support of $H_{0}(\boldsymbol{\omega})$ are glued in different manners. Notice that in the twoparallelogram filter banks, the analysis filters have only two passbands; when the filters have real coefficients, the location of one passband will determine the location of the other passband. This is not true in the four-parallelogram filter bank case, as we can see from Fig. 7. The preceding construction indicates that for a given decimation matrix $M$, the support configuration is determined by the following two steps.

Step 1) Choose $L$ and, hence, the parallelogram prototype.

Step 2) Shift the parallelogram prototype properly, and combine four shifted copies to obtain the support for each analysis filter.

We will construct various types of configurations for the fourparallelogram filter banks and discuss permissibility of these configurations.

It turns out that permissibility is only possible for a special class of four-parallelogram filter banks: the simplistic fourparallelogram filter banks. We will see that in permissible simplistic four-parallelogram filter banks, the decimation matrix $M$ is necessarily of the form $Q \mathbb{A}$, where $Q$ is an integer matrix with $|Q|=1$ or 2 , and $\Lambda$ is a diagonal integer matrix. This gives rise to the implementation of simplistic fourparallelogram filter banks of low design cost. In particular, when $|Q|=1$, simplistic four-parallelogram filter banks can be implemented by using a separable filter bank with decimation matrix $\Lambda$ followed by a so-called unimodular transformation [8]. When $|Q|=2$, the desired configuration can be achieved by concatenating a separable 2-D filter banks with a 2-D twochannel filter bank in the form of a tree. In the companion paper [1], we discuss cosine-modulated implementation of two-parallelogram filter banks. For four-parallelogram filter banks, we will not do it because attractive implementation can be obtained with little loss of generality, as we will show.

\section{Paper Outline}

All notations and conventions are precisely as in [1]. The sections are organized as follows. In Section II, we will consider a special class of four-parallelogram filter banks: the simplistic four-parallelogram filter banks. For a given decimation matrix $\boldsymbol{M}$, we say a four-parallelogram filter bank is simplistic if the support of the lowpass analysis filter is the parallelogram $S P D\left(\pi \boldsymbol{M}^{-T}\right)$. The other filters in the simplistic filter banks will be construcuted such that the configuration of the filter bank is permissible. We will see that in this case, the decimation matrix needs to be property constrained. In Section III, we make a connection between permissible simplistic fourparallelogram filter banks to other existing filter banks. Via this connection, permissible simplistic four-parallelogram filter banks can be achieved by cascading filter banks of low design cost. Other types of four-parallelogram filter banks (other than the simplistic four-parallelogram filter banks) are studied in Section IV.

\section{The Simplistic Four-Parallelogram Filter Banks}

Consider the filter bank with decimation matrix $M$ in Fig. 2 . In this section, we consider simplistic four-parallelogram filter banks: a special type of four-parallelogram filter banks that are completely characterized by the support of the lowpass analysis filter $H_{0}(\boldsymbol{\omega})$. A four-parallelogram filter bank with decimation matrix $M$ is called simplistic if the support of $H_{0}(\omega)$ is $S P D\left(\pi M^{-T}\right)$. Recall that in a separable filter bank with diagonal decimation matrix $\Lambda$, the support of the lowpass analysis filter is $\operatorname{SPD}\left(\pi \Lambda^{-T}\right)$. Therefore, the lowpass analysis filter in simplistic filter banks is the natural nonseparable generalization of lowpass analysis filter in separable filter bank.

We can verify that in simplistic filter banks, the matrix $L$ is

$$
L=\left[\begin{array}{ll}
2 & 0 \\
0 & 2
\end{array}\right] \text {. }
$$

The matrix $\boldsymbol{N}$ that determines the parallelogram prototype $\operatorname{SPD}\left(\pi \boldsymbol{N}^{-T}\right)$ is $\boldsymbol{N}=2 \boldsymbol{M}$. For example,

$$
\text { let } \boldsymbol{M}=\left[\begin{array}{cc}
8 & -5 \\
-4 & 5
\end{array}\right], \text { then } \boldsymbol{N}=2\left[\begin{array}{cc}
8 & -5 \\
-4 & 5
\end{array}\right] \text {. }
$$




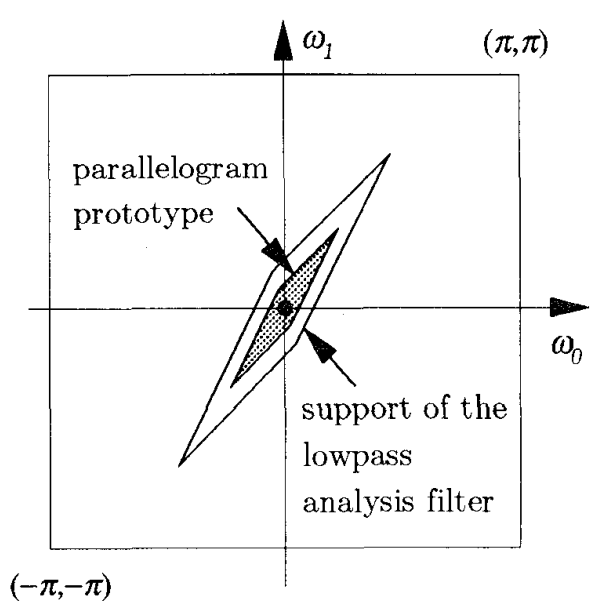

Fig. 8. Simplistic four-parallelogram filter bank. The parallelogram prototype $S P D\left(\pi N^{-T}\right)$ and the support of the lowpass analysis filter $S P D\left(\pi M^{-T}\right)$.

The parallelogram prototype $S P D\left(\pi \boldsymbol{N}^{-T}\right)$ and the support of the lowpass analysis filter $S P D\left(\pi M^{-T}\right)$ are as shown in Fig. 8.

Frequency Normalization: All the frequency planes in previous figures are shown with $\omega_{0}$ and $\omega_{1}$ as two axes, e.g., Fig. 8. For the convenience of illustration, we will use a new set of axes. On the new frequency plane, the parallelogram prototype would appear the same for any chosen $N$. More specifically, we will normalize the frequency plane by $2 \pi N^{-T}$; we define

$$
\boldsymbol{\nu}=\left[\begin{array}{l}
\nu_{0} \\
\nu_{1}
\end{array}\right]=\frac{1}{2 \pi} \boldsymbol{N}^{T}\left[\begin{array}{l}
\omega_{0} \\
\omega_{1}
\end{array}\right] .
$$

and use $\nu_{0}$ and $\nu_{1}$ as the two axes. On the normalized frequency plane, the parallelogram prototype will always appear as the square $S P D(0.5 I)$ (Fig. 9). In the simplistic filter bank case, the support of $H_{0}(\boldsymbol{\omega})$ becomes $S P D(\boldsymbol{I})$, as shown in Fig. 9. In Fig. 9, the notation $S_{\left(k_{0}, k_{1}\right)}$ denotes the square

$$
S P D(0.5 I)+\left[\begin{array}{l}
k_{0}+0.5 \\
k_{1}+0.5
\end{array}\right]
$$

where the vector subscript of $S$ should be interpreted modulo $N^{T}$. The support of $H_{0}(\boldsymbol{\omega})$ is the union of the four squares

$$
S_{(0,0)}, S_{(-1,0)}, S_{(0,-1)} ; \quad \text { and } S_{(-1,-1)}
$$

Notice that to have real-coefficient filters, whenever $S_{\left(k_{0}, k_{1}\right)}$ belongs to a certain analysis filter, $S_{\left(-k_{0}-1,-k_{1}-1\right)}$ must be part of the same analysis filter. We will call $\left(S_{\left(k_{0}, k_{1}\right)}, S_{\left(-k_{0}-1,-k_{1}-1\right)}\right)$ a conjugate pair. The support of each analysis filter consists of two conjugate pairs.

Necessary Condition for Permissibility: Thus far, only the support of the lowpass analysis filter is determined. As permissibility is jointly determined by all the analysis filters, the supports of the analysis filters in other subbands have to be constrained properly. We would like to construct the other filters such that the configuration has edge and vertex permissibility at the same time. With this premise, we can derive the following necessary condition.

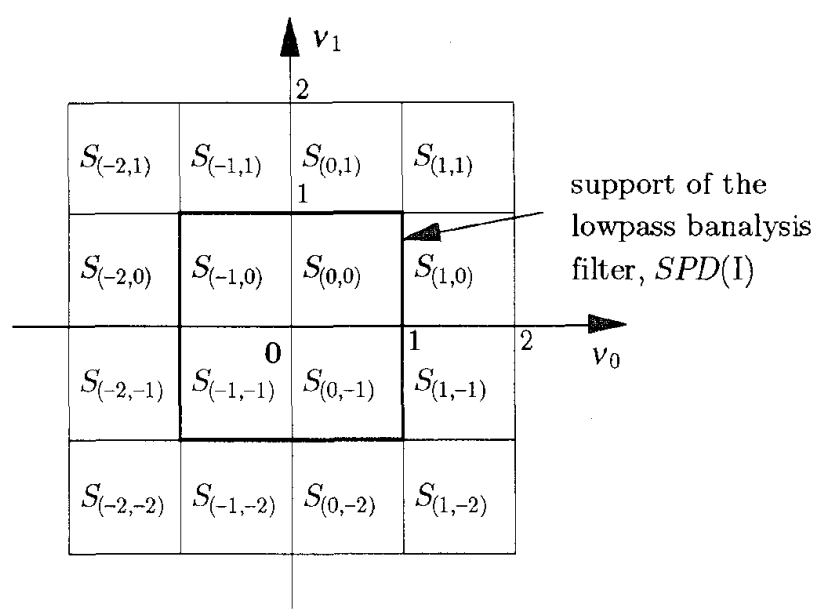

Fig. 9. Simplistic four-parallelogram filter bank. The support of the lowpass analysis filter.

Proposition 2.1: If a simplistic four-parallelogram filter bank is permissible, the supports of the other analysis filters $H_{m}(\boldsymbol{\omega})$ must contain

the conjugate pair $\left(S_{\left(k_{0}, k_{1}\right)}, S_{\left(-k_{0}-1,-k_{1}-1\right)}\right)$,

$$
\text { and the conjugate pair }\left(S_{\left(-k_{0}-1, k_{1}\right)}, S_{\left(k_{0},-k_{1}-1\right)}\right) \text {, . }
$$

Corollary 2.1: The condition in Proposition 2.1 in terms implies that decimation matrix $M$ is restricted to the following form:

$$
M=Q \Lambda
$$

where $Q$ is an integer matrix with $|Q|=1$ or 2 , and $\boldsymbol{\Lambda}$ is a diagonal integer matrix.

Proposition 2.1 and Corollary 2.1 will be shown in Sections II-A adn B, respectively.

\section{A. Edge and Vertex Permissible Simplistic Four-Parallelogram Filter Banks}

Recall that the $\boldsymbol{M}$-fold decimated and expanded version of $H_{0}(\boldsymbol{\omega})$ contains $|\boldsymbol{M}|$ copies, which are shifted copies of $H_{0}(\boldsymbol{\omega})$ by

$$
2 \pi \boldsymbol{M}^{-T} \boldsymbol{m}, \boldsymbol{m} \in \mathcal{N}\left(\boldsymbol{M}^{T}\right) .
$$

With axes normalization, the images of passband $S_{k}$ are at

$$
S_{\boldsymbol{k}+2 \boldsymbol{m}}, \quad \boldsymbol{m} \in \mathcal{N}\left(\boldsymbol{M}^{T}\right), \quad \boldsymbol{m} \neq \mathbf{0} .
$$

Fig. 10 illustrates the locations of the images of $S_{k}$. The images of $S_{k}$ are neither edge adjacent nor vertex adjacent to $S_{k}$. It follows from (4) that a particular $S_{n}$ is occupied by an image of $S_{\boldsymbol{k}}$ if $\frac{1}{2}(\boldsymbol{n}-\boldsymbol{k})$ is an integer vector. Furthermore, the aliasing resulting from the image at $S_{n}$ contributes to the aliasing transfer function $A_{(1 / 2)(\boldsymbol{n}-\boldsymbol{k})}(\boldsymbol{\omega})$ (see Appendix A of [1] for the definition of aliasing transfer functions). In the equations to follow, the vector subscript $n$ of $S_{n}$ should 


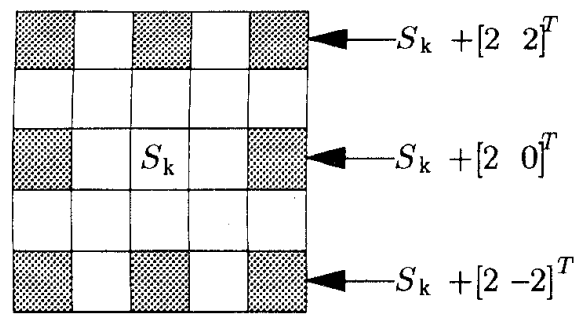

Fig. 10. Location of the images of $S_{k}$.

be interpreted modulo $\boldsymbol{N}^{T}$, whereas the vector subscript $\boldsymbol{k}$ of $A_{k}(\omega)$ should be interpreted modulo $M^{T}$.

From Fig. 10, we see that three images of $S_{(0,0)}$ will be at $S_{(-2,0)}, S_{(0,-2)}$ and $S_{(-2,-2)}$, as shown in Fig. 11. The images at $S_{(-2,0)}, S_{(0,-2)}$ are edge adjacent to $H_{0}(\boldsymbol{\omega})$ and result in edge aliasing, whereas the image at $S_{(-2,-2)}$ is vertex adjacent to $H_{0}(\boldsymbol{\omega})$ and results in vertex aliasing. We discuss, respectively, the cancelation of aliasing on account of the three images mentioned above. We will argue that due to the presence of the images at $S_{(-2,0)}$ and $S_{(0,-2)}$, the supports of two other analysis filters will be determined.

1) The Image at $S_{(-2,0)}$ : From Fig. 11, we see that this image is edge adjacent to $S_{(-1,0)}$. The aliasing term contributes to the aliasing transfer function $A_{(-1,0)}(\omega)$. Let the support of the analysis filter $H_{1}(\omega)$ contain $S_{(-2,0)}$. Then, this is the only subband that can provide an aliasing term to cancel the aliasing error in the first subband. To be able to contribute to the aliasing transfer function $A_{(-1,0)}(\boldsymbol{\omega})$, the support of $H_{1}(\boldsymbol{\omega})$ must contain $S_{(1,0)}$. As the analysis filters have real coefficients, the support of $H_{1}(\boldsymbol{\omega})$ must contain the conjugate pairs $\left(S_{(1,0)}, S_{(-2,-1)}\right)$ and $\left(S_{(-2,0)}, S_{(1,-1)}\right)$ (Fig. 9). Summarizing, the support of $H_{1}(\boldsymbol{\omega})$ is the union of

$$
S_{(1,0)}, S_{(-2,-1)}, S_{(-2,0)}, \quad \text { and } \quad S_{(1,-1)}
$$

as shown in Fig. 12.

2) The Image at $S_{(0,-2)}$ : Similarly to the previous case, this image is edge adjacent to $S_{(0,-1)}$ and creates an aliasing term that contributes to the aliasing transfer function $A_{(0,-1)}(\omega)$. Let the support of the analysis filter $H_{2}(\omega)$ contain $S_{(0,-2)}$. To be able to cancel the edge aliasing error in the zeroth subband, the support of $H_{2}(\boldsymbol{\omega})$ must also contain $S_{(0,1)}$. As the analysis filters are real-coefficient, the support of $H_{2}(\omega)$ is the union of

$$
S_{(0,1)}, S_{(-1,-2)}, S_{(0,-2)}, \quad \text { and } \quad S_{(-1,1)},
$$

as shown in Fig. 12.

3) The Image at $S_{(-2,-2)}$ : This image is vertex adjacent to $S_{(-1,-1)}$. This will result in aliasing error that contributes to $A_{(-1,-1)}(\boldsymbol{\omega})$. Similarly, it is necessary that $S_{(-2,-2)}$ and $S_{(-1,-1)}$ are parts of the same analysis filter. As the analysis filters are real-coefficient, $S_{(-2,-2)}$ and $S_{(-1,-1)}$ always belong to the same analysis filter.

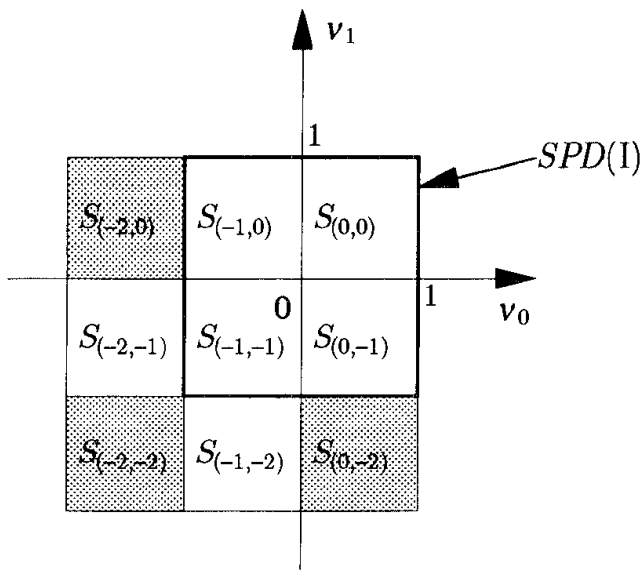

Fig. 11. Simplistic four-parallelogram filter bank. The image of $S_{(0,0)}$.

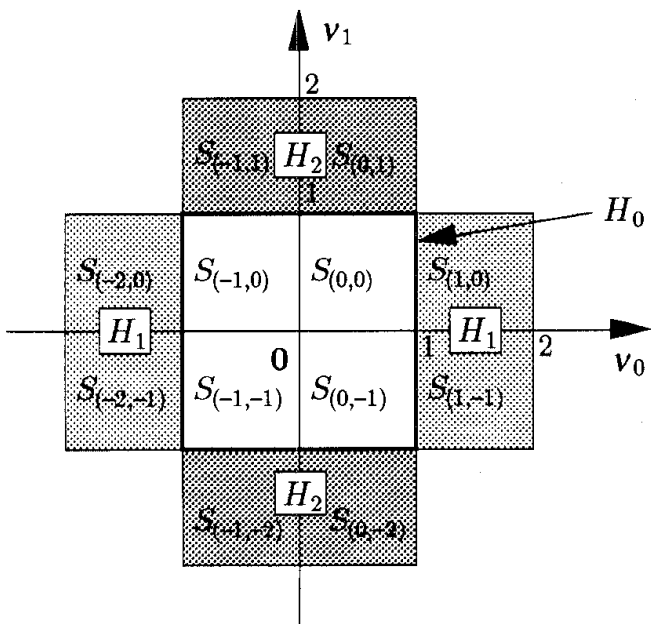

Fig. 12. Second and third analysis filters in the simplistic four-parallelogram filter bank.

We can verify that the cancelation of aliasing caused by the images of $S_{(0,-1)}, S_{(-1,0)}$ and $S_{(-1,-1)}$ require the same choice of support for the second and third subbands. Repeating the same argument, we conclude that for the purpose of alias cancelation or support permissibility the analysis filters are necessarily of the form in (2).

In a typical 1-D $M$-channel filter bank, each analysis filter has total bandwidth $2 \pi / M$. In the $2-\mathrm{D}$ case, total bandwidth should be interpreted as total area of spectral occupancy. When the filters are not constructed properly, some filters would have less area than others. The following result shows that this situation can be avoided.

Proposition 2.2: When the supports of the analysis filters are chosen according to (2), all analysis filters have the same area of spectral occupancy.

Proof: We only need to show that the four squares are distinct under modulo $\boldsymbol{N}^{T}$. For this, we will show that $S_{\left(k_{0}, k_{1}\right)}$ is not the same as the other three terms in (2). Suppose that $S_{\left(k_{0}, k_{1}\right)}$ and $S_{\left(-k_{0}-1, k_{1}\right)}$ are identical for some $k_{0}$ and $k_{1}$. Then, $\left[\begin{array}{l}k_{0} \\ k_{1}\end{array}\right]=\left[\begin{array}{c}-k_{0}-1 \\ k_{1}\end{array}\right] \bmod \boldsymbol{N}^{T}$, which implies $\left[\begin{array}{c}2 k_{0}+1 \\ 0\end{array}\right]=\mathbf{0}$ $\bmod N^{T}$, i.e., $\left[\begin{array}{c}2 k_{0}+1 \\ 0\end{array}\right]$ is on the lattice of $N^{T}$. However, 


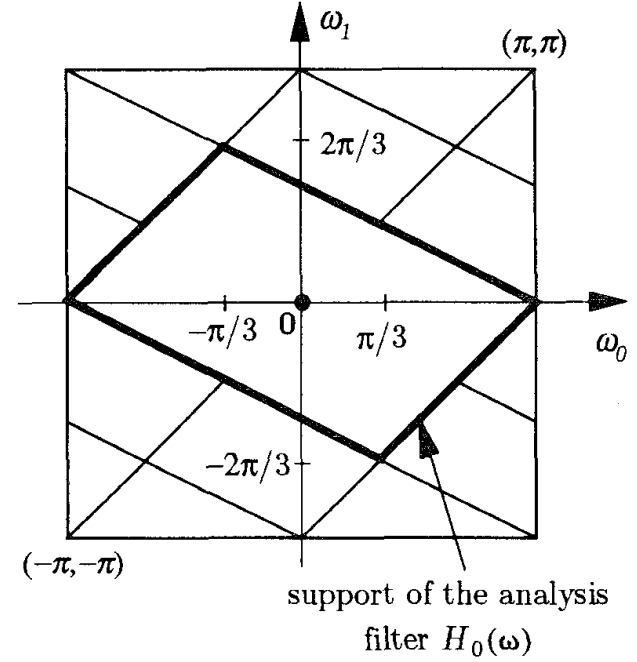

(a)

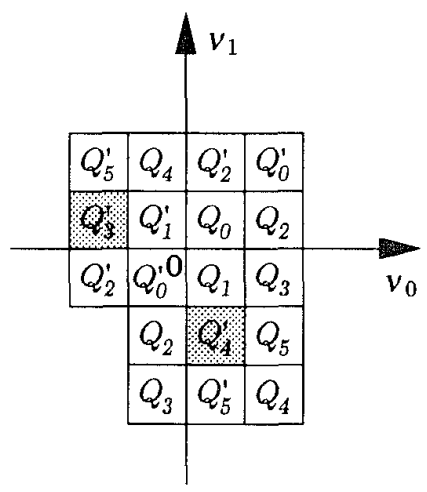

(b)

Fig. 13. Example 2.1. (a) Support of the zeroth analysis filter. (b) Pertaining to the illustration of alias cancelation.

$\boldsymbol{N}^{T}=2 \boldsymbol{M}^{T}$ and $\left[\begin{array}{c}2 k_{0}+1 \\ 0\end{array}\right]$ can not be a vector of $\operatorname{LAT}\left(\boldsymbol{N}^{T}\right)$. Therefore, $S_{\left(k_{0}, k_{1}\right)}$ and $S_{\left(-k_{0}-1, k_{1}\right)}$ are distinct $\forall k_{0}, k_{1}$. In a similar manner, we can show that $S_{\left(k_{0}, k_{1}\right)}$ and $S_{\left(k_{0},-k_{1}-1\right)}$ are distinct and that $S_{\left(k_{0}, k_{1}\right)}$ and $S_{\left(-k_{0}-1,-k_{1}-1\right)}$ are distinct $\forall k_{0}, k_{1}$. as well.

\section{B. Proof of Corollary 2.1}

We first show that support permissibility will imply that the matrix $K$ given by

$$
\boldsymbol{K}=\boldsymbol{M}^{-T} \underbrace{\left[\begin{array}{cc}
1 & 0 \\
0 & -1
\end{array}\right]}_{\boldsymbol{\Lambda}_{0}} \boldsymbol{M}^{-T}
$$

is an integer matrix, which in turn will give us (3).

By (2), the analysis filter that contains the pair $\left(S_{\left(k_{0}, k_{1}\right)}, S_{\left(-k_{0}-1,-k_{1}-1\right)}\right)$ should also contain $\left(S_{\left(-k_{0}-1, k_{1}\right)}, S_{\left(k_{0},-k_{1}-1\right)}\right)$. Suppose $\left[\begin{array}{l}k_{0} \\ k_{1}\end{array}\right]=\left[\begin{array}{l}k_{0}^{\prime} \\ k_{1}^{\prime}\end{array}\right] \bmod$ $N^{T}$. Then,

$$
\left[\begin{array}{l}
k_{0}-k_{0}^{\prime} \\
k_{1}-k_{1}^{\prime}
\end{array}\right]=0 \bmod N^{T}
$$

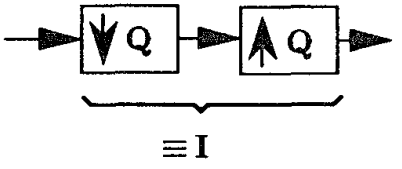

Fig. 14. Cascade of a unimodular decimator $Q$ and a unimodular expander $Q$

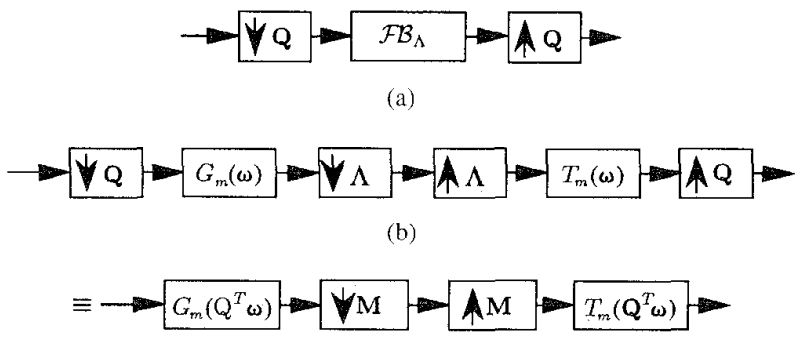

(c)

Fig. 15. Pertaining to the illustration of the unimodular transformation.

The conjugate pair $\left(S_{\left(k_{0}, k_{1}\right)}, S_{\left(-k_{0}-1,-k_{1}-1\right)}\right)$ should be the same as the pair $\left(S_{\left(k_{0}^{\prime}, k_{1}^{\prime}\right)}, S_{\left(-k_{0}^{\prime}-1,-k_{1}^{\prime}-1\right)}\right)$. It follows that $\left(S_{\left(-k_{0}-1, k_{1}\right)}, S_{\left(k_{0},-k_{3}-1\right)}\right)$ is the same as the pair $\left(S_{\left(-k_{0}^{\prime}-1, k_{1}^{\prime}\right)}, S_{\left(k_{0}^{\prime},-k_{1}^{\prime}-1\right)}\right)$. With $\boldsymbol{N}=2 \boldsymbol{M}$, we can verify that $\left[-k_{0}-1, k_{1}\right]^{T} \neq\left[k_{0}^{\prime},-k_{1}^{\prime}-1\right]^{T} \bmod N^{T}$, for all $k_{0}$ and $k_{1}$. Therefore, we have $S_{\left(-k_{0}-1, k_{1}\right)}=S_{\left(-k_{0}^{\prime}-1, k_{1}^{\prime}\right)}$, and hence,

$$
\left[\begin{array}{l}
k_{0}^{\prime}-k_{0} \\
k_{1}-k_{1}^{\prime}
\end{array}\right]=0 \bmod N^{T} .
$$

Combining (6) and (7), we have $\Lambda_{0} N^{T} d=0 \bmod N^{T}, \forall d \in$ $\mathcal{Z}^{2}$, where $\Lambda_{0}$ is as defined in (5), and $\mathcal{Z}^{2}$ is the set of all $2 \times 1$ integer vectors. Let $d_{0}=\left[\begin{array}{ll}1 & 0\end{array}\right]^{T}$ and $\boldsymbol{d}_{1}=\left[\begin{array}{ll}0 & 1\end{array}\right]^{T}$; then,

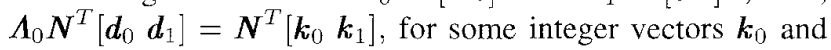

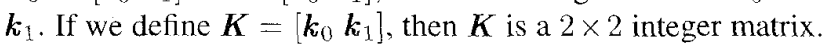
Since $\left[\boldsymbol{d}_{0} \boldsymbol{d}_{1}\right]$ is the $2 \times 2$ identity matrix, and $N=2 M$, we have $\boldsymbol{\Lambda}_{0} \boldsymbol{M}^{T}=\boldsymbol{M}^{T} \boldsymbol{K}$, and we conclude that $\boldsymbol{K}=\boldsymbol{M}^{T} \boldsymbol{\Lambda}_{0} \boldsymbol{M}^{T}$ is an integer matrix. In fact, as $\left|\boldsymbol{M}^{-T} \boldsymbol{\Lambda}_{0} \boldsymbol{M}^{T}\right|=1, \boldsymbol{K}$ is unimodular.

Let the decimation matrix $M$ have Smith form $\boldsymbol{M}=$ $\boldsymbol{U}_{s} \boldsymbol{\Lambda}_{s} \boldsymbol{V}_{s}$, where $\left[\boldsymbol{\Lambda}_{s}\right]_{00}=\beta$ and $\left[\boldsymbol{\Lambda}_{s}\right]_{11}=\alpha \beta$ (see Appendix A of [1] for the definition of the Smith form of integer matrices). Let $V_{s}^{T}=\left[\begin{array}{ll}v_{0} & v_{10} \\ v_{01} & v_{11}\end{array}\right]$; then,

$$
\boldsymbol{K}=\left|\boldsymbol{V}_{s}^{T}\right|\left[\begin{array}{cc}
v_{00} v_{11}+v_{10} v_{01} & 2 \alpha v_{01} v_{11} \\
-\frac{2}{\alpha} v_{00} v_{10} & -v_{00} v_{11}-v_{10} v_{01}
\end{array}\right]
$$

The matrix $K$ is unimodular if $\alpha$ divides $2 v_{00} v_{10}$.

1) $\alpha$ is odd: Suppose $\alpha=\alpha_{1} \alpha_{2}, \alpha_{1}$ divides $v_{00}$, and $\alpha_{2}$ divides $v_{10}$. Then,

$$
\boldsymbol{M}=\underbrace{\boldsymbol{U}_{s}\left[\begin{array}{cc}
v_{00} / \alpha_{1} & v_{10} / \alpha_{2} \\
\alpha_{2} v_{01} & \alpha_{1} v_{11}
\end{array}\right]}_{\boldsymbol{Q}} \beta \underbrace{\beta\left[\begin{array}{cc}
\alpha_{1} & 0 \\
0 & \alpha_{2}
\end{array}\right]}_{\boldsymbol{\lambda}}
$$

where the matrix $Q$ has $|Q|=1$. 


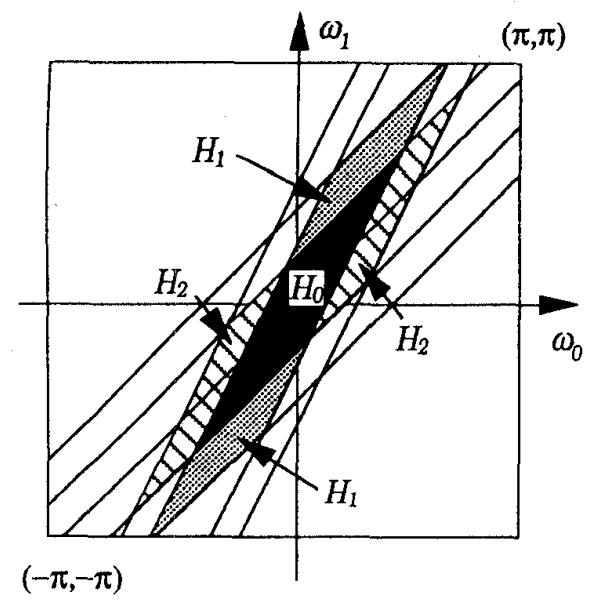

(a)

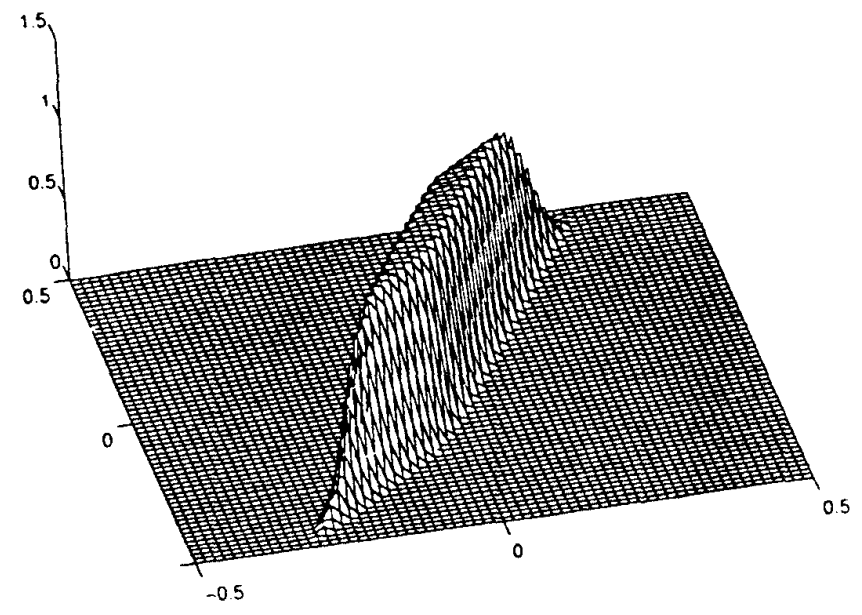

(b)

Fig. 16. Example 3.1. Simplistic four-parallelogram filter bank. (a) Spectral support of the analysis filters and (b) the magnitude response of the lowpass analysis filter with frequency normalized by $2 \pi$.

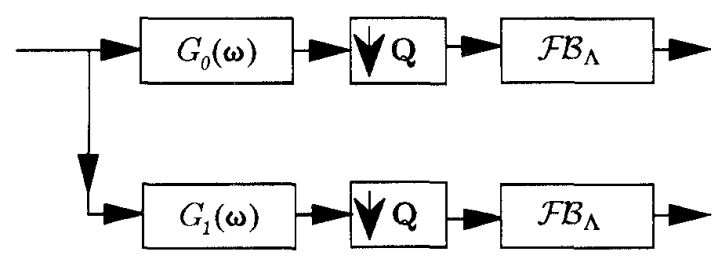

Fig. 17. Two-level tree structured filter bank. The first level is a 2-D two-channel filter bank with decimation matrix $Q$, and the second level is a 2-D separable filter bank with decimation matrix $A$.

2) $\alpha$ is even: Suppose $\alpha=2 \alpha_{1} \alpha_{2}, \alpha_{1}$ divides $v_{00}$, and $\alpha_{2}$ divides $v_{10}$. Then,

$$
\boldsymbol{M}=\underbrace{\boldsymbol{U}_{s}\left[\begin{array}{cc}
1 & 0 \\
0 & 2
\end{array}\right]\left[\begin{array}{cc}
v_{00} / \alpha_{1} & v_{10} / \alpha_{2} \\
\alpha_{2} v_{01} & \alpha_{1} v_{11}
\end{array}\right]}_{\boldsymbol{Q}} \underbrace{\beta\left[\begin{array}{cc}
\alpha_{1} & 0 \\
0 & \alpha_{2}
\end{array}\right]}_{\boldsymbol{\Lambda}_{2}}
$$

where $Q$ has $|Q|=2$. Notice that in the special case of the two-channel 2-D filter bank $\alpha=2$ and $\beta=1$, the matrix $K$ is always unimodular, and (7) is satisfied.

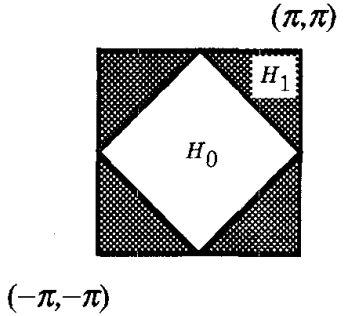

(a)

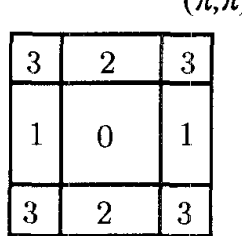

$(-\pi,-\pi)$

(b)

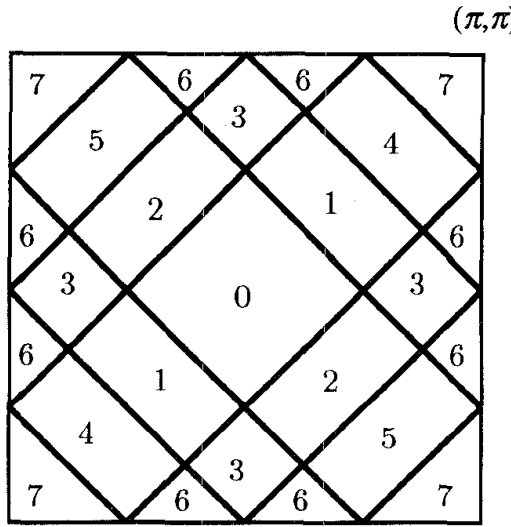

$(-\pi,-\pi)$

(c)

Fig. 18. Example 3.2. The simplistic four-parallelogram filter bank. (a) Two-channel diamond filter bank. (b) Supports of the analysis filters in a four-channel separable filter bank (with the support of the $k$ th filter denoted by $k$ ). (c) Supports of the eight analysis filters in the overall system. The support of the $k$ th analysis filter is denoted by $k$.

Example 2.1-Decimation Matrix $\boldsymbol{M}$ Not Satisfying (3): This example illustrates that some edge aliasing errors will remain uncancelled when (2) is not satisfied. Consider the decimation matrix

$$
\boldsymbol{M}=\left[\begin{array}{cc}
1 & 1 \\
-1 & 2
\end{array}\right]
$$

which is not of the form in (3), and hence, the analysis filters cannot be of the form in (2). The supports of the analysis filters consist of four parallelograms, each a shifted version of $S P D\left(\pi N^{-T}\right)$, where $N=2\left[\begin{array}{cc}1 & 1 \\ -1 & 2\end{array}\right]$. The support of the lowpass analysis filter $H_{0}(\boldsymbol{\omega})$ is as shown in Fig. 13(a). With axis normalization the support of $H_{0}(\boldsymbol{\omega})$ becomes $S P D(\boldsymbol{I})$, as shown in Fig. 13(b). We have labeled all the cells in Fig. 13(b) 


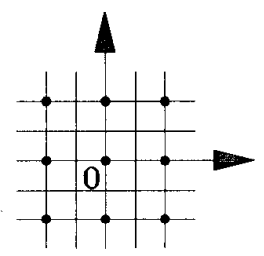

(a)

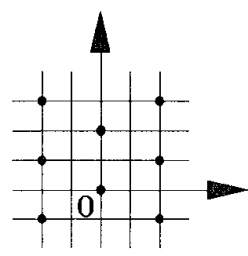

(b)

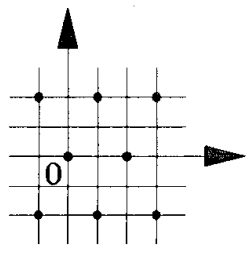

(c)

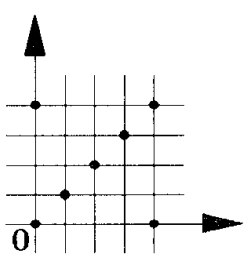

(d)

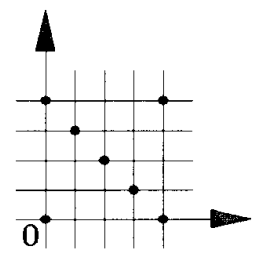

(e)

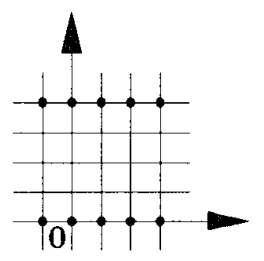

(f)

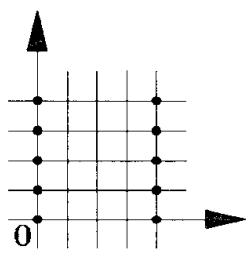

(g)

Fig. 19. Possible lattices of integer matrix $L^{T}$, where $L$ has $|L|=4$.

by $Q_{k}$ and $Q_{k}^{\prime}$ with $\left(Q_{k}, Q_{k}^{\prime}\right)$ denoting a conjugate pair. As $|\boldsymbol{N}|=12$, there are total of six conjugate pairs $\left(Q_{k}, Q_{k}^{\prime}\right)$, for $k=0,1, \cdots, 5$. The lowpass filter $H_{0}(\boldsymbol{\omega})$ contains the pair $\left(Q_{0}, Q_{0}^{\prime}\right)$ and the pair $\left(Q_{1}, Q_{1}^{\prime}\right)$. The two images of $Q_{0}$ are at $Q_{3}^{\prime}$ and $Q_{4}^{\prime}$. The image at $Q_{3}^{\prime}$ is edge adjacent to $Q_{1}^{\prime}$. To cancel the aliasing from this image, it is necessary that the analysis filter contain both $Q_{3}^{\prime}$ and $Q_{2}$. Let this analysis filter be $H_{1}(\boldsymbol{\omega})$. Then, $H_{1}(\boldsymbol{\omega})$ consists of the pairs $\left(Q_{2}, Q_{2}^{\prime}\right)$ and the pair $\left(Q_{3}, Q_{3}^{\prime}\right)$. In this case, the two pairs left for the last analysis filter $H_{2}(\boldsymbol{\omega})$ are $\left(Q_{4}, Q_{4}^{\prime}\right)$ and $\left(Q_{5}, Q_{5}^{\prime}\right)$. However, the image of $Q_{0}$ at $Q_{4}^{\prime}$ is edge adjacent to $Q_{1}$ and results in edgebased aliasing error. Cancelation of this aliasing requires that $Q_{4}^{\prime}$ and $Q_{2}^{\prime}$ belong to the same analysis filter, i.e., $\left(Q_{2}, Q_{2}^{\prime}\right)$ and $\left(Q_{4}, Q_{4}^{\prime}\right)$ belong to the same analysis filter. As the support of $H_{2}(\boldsymbol{\omega})$ consists of $\left(Q_{4}, Q_{4}^{\prime}\right)$ and $\left(Q_{5}, Q_{5}^{\prime}\right)$, this aliasing cannot be cancelled.

\section{DESIGN OF THE SimplistiC Four-PARALLELOGRAM FILTER BANKS}

The results presented in the previous section show that for permissibility of simplistic four-parallelogram filter banks, the decimation matrix $M$ has the special form $M=Q \Lambda$, where $Q$ is an integer matrix with $|Q|=1$ or 2 , and $\boldsymbol{\Lambda}$ is a diagonal integer matrix. As a result, the support described in (2) can always be obtained by cascading systems of low design cost. In particular, when the matrix $Q$ has $|\boldsymbol{Q}|=$ 1 , the support configurations can be obtained by designing two 1-D perfect reconstruction filter banks and performing a unimodular transformation [8]. When the matrix $Q$ has $|Q|=$ 2 , the desired configuration can be achieved by concatenating a separable 2-D filter banks with a 2-D two-channel filter bank in the form of a tree structure. We now explain both of these in greater detail.

Case $1-|Q|=1$

Consider a 2-D $|\boldsymbol{M}|$-channel perfect reconstruction filter bank obtained by cascading two 1-D filter banks using tree structure. The first and second levels of the tree are 1-D filter banks with $[\boldsymbol{\Lambda}]_{00}$ channels and $[\boldsymbol{\Lambda}]_{11}$. The overall system, having decimation matrix $\Lambda$, will be denoted by $\mathcal{F B} \Lambda$. Let the analysis and synthesis filters of $\mathcal{F} B_{\Lambda}$ be defined as $G_{m}(\boldsymbol{\omega})$ and $T_{m}(\boldsymbol{\omega})$, respectively. Then, the support of $G_{m}(\boldsymbol{\omega})$ is the union of four rectangles of $\operatorname{SPD}\left(\pi \frac{1}{2} \boldsymbol{\Lambda}^{-\mathbf{1}}\right)$. Recall that a unimodular decimator or a unimodular expander only permutes the input, and the cascade of a unimodular decimator $Q$ followed by a unimodular expander $Q$ is an identity system; see Fig. 14. Now, if we insert a unimodular decimator $Q$ before $\mathcal{F B} A$ and insert a unimodular expander $Q$ after $\mathcal{F B}_{\Lambda}$, the new system (Fig. 15(a)) remains a perfect reconstruction system. This is equivalent to inserting a decimator $Q$ before each analysis filter and an expander $Q$ after each synthesis filter (Fig. 15(b)). We can redraw Fig. 15(b) as Fig. 15(c). Denote the new filter bank with decimation matrix $M=Q \Lambda$ by $\mathcal{F B} M$, which will be called the unimodular transformation of $\mathcal{F B} A$ by $Q$. The analysis and synthesis filters of $\mathcal{F B}^{M}$ are $G_{m}\left(\boldsymbol{Q}^{T} \boldsymbol{\omega}\right)$ and $T_{k}\left(\boldsymbol{Q}^{T} \boldsymbol{\omega}\right)$. Observe that the support of $G_{m}\left(\boldsymbol{Q}^{T} \boldsymbol{\omega}\right)$ is the union of four shifted copies of $S P D\left(\pi \frac{1}{2} \boldsymbol{Q}^{-T} \boldsymbol{\Lambda}^{-1}\right)$. One can verify that the filter bank $\mathcal{F B}_{M}$ with analysis filters $H_{m}(\boldsymbol{\omega})$ and synthesis filters $F_{m}(\boldsymbol{\omega})$ given by

$$
H_{m}(\boldsymbol{\omega})=G_{m}\left(\boldsymbol{Q}^{T} \boldsymbol{\omega}\right), \quad F_{m}(\boldsymbol{\omega})=T_{m}\left(\boldsymbol{Q}^{T} \boldsymbol{\omega}\right)
$$

is a simplistic four-parallelogram filter bank. This result is identical to those found earlier in [8].

Example 3.1-Simplistic Four-Parallelogram Filter Banks: Consider a 20-channel filter bank with decimation matrix

$$
M=\left[\begin{array}{cc}
8 & -5 \\
-4 & 5
\end{array}\right]
$$

which can be factorized as $\left[\begin{array}{cc}2 & -1 \\ -1 & 1\end{array}\right]\left[\begin{array}{ll}4 & 0 \\ 0 & 5\end{array}\right]$. The matrix $\boldsymbol{M}$ has the form in case 1 of (2). Design a separable filter bank by concatenating a 1-D four-channel filter bank and a 1D five-channel filter bank. Using the substitution in (8), the 


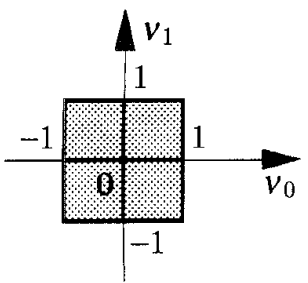

(a)

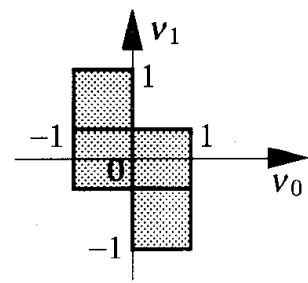

(b)

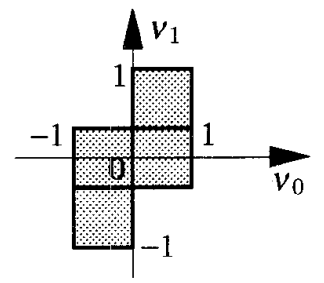

(c)

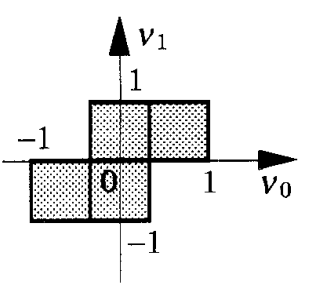

(d)

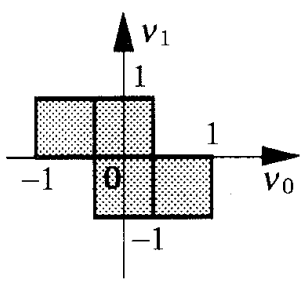

(e)

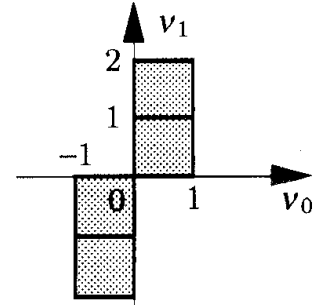

(f)

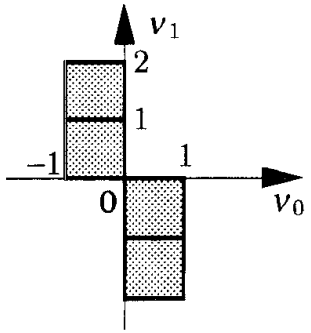

(g)

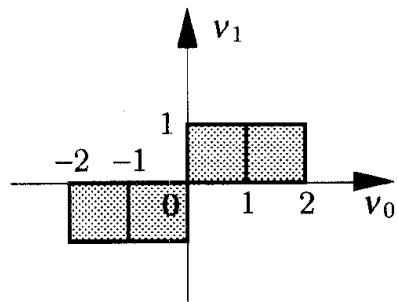

(h)

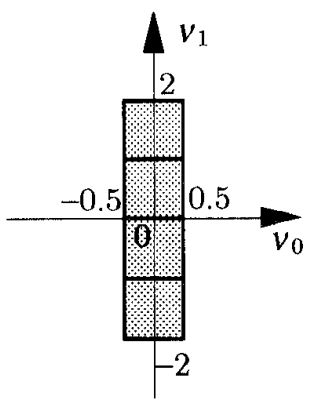

(k)

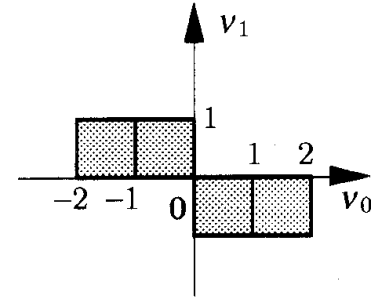

(i)

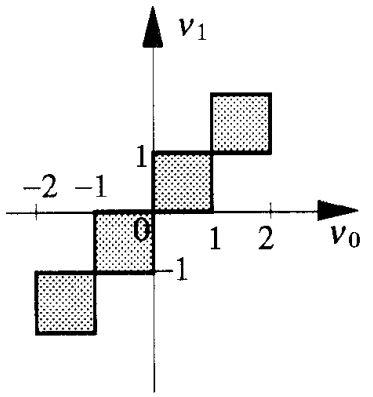

(1)

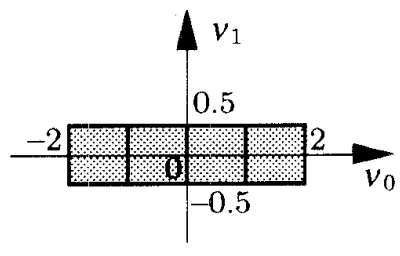

(j)

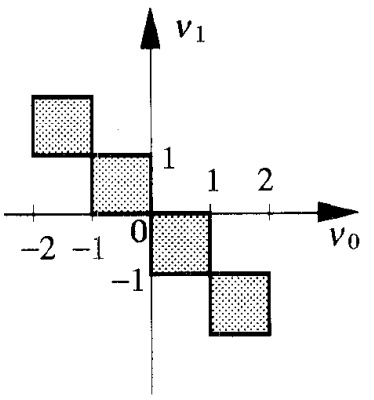

(m)

Fig. 20. Possible supports of the lowpass analysis filter.

new nonseparable filter bank has the desired configuration as described in (2). The parallelogram prototype is as shown in Fig. 8. Fig. 16(a) shows the support configuration of the the analysis filters. Fig. 16(b) shows the magnitude response of the lowpass analysis filter.

Case $2-|Q|=2$

Consider a tree structured filter bank with two levels in Fig. 17. The first level of the tree is a two-channel filter bank with decimation matrix $Q$. The lowpass analysis filter of the two-channel system has support $S P D\left(\pi Q^{-T}\right)$. For the second level of the tree, we use a separable $|\boldsymbol{\Lambda}|$-channel filter bank $\left(\mathcal{F B} \mathcal{B}_{A}\right)$ that is obtained by cascading two 1-D filter banks of $[\Lambda]_{00}$ channels and $[\Lambda]_{11}$ channels in the form of a tree.

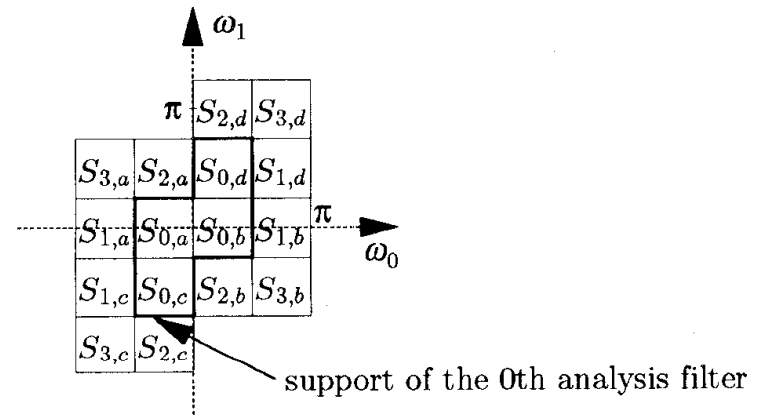

Fig. 21. Example 4.1. The support configuration of a permissible four-parallelogram filter bank that is not simplistic.

Then, the overall filter bank has the desired configuration of four-parallelogram filter banks. 


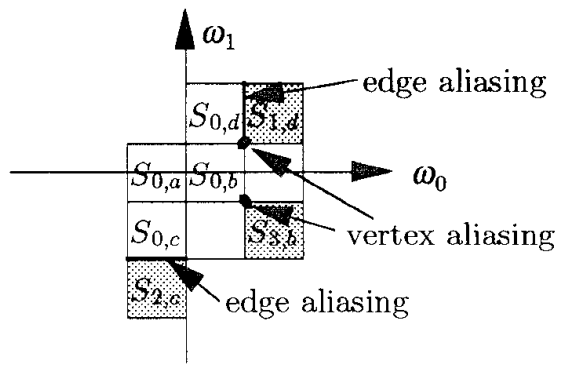

(a)

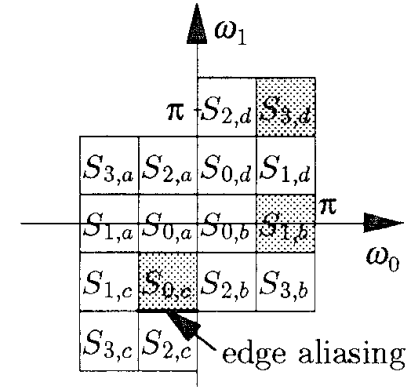

(b)

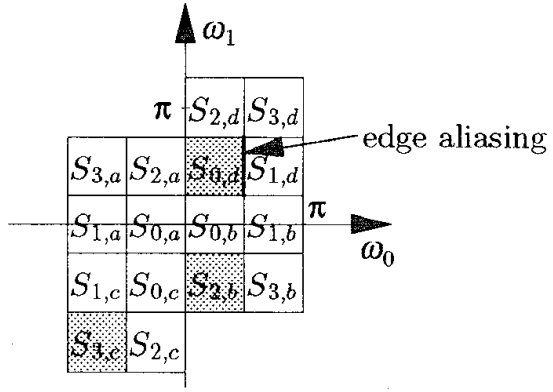

(c)

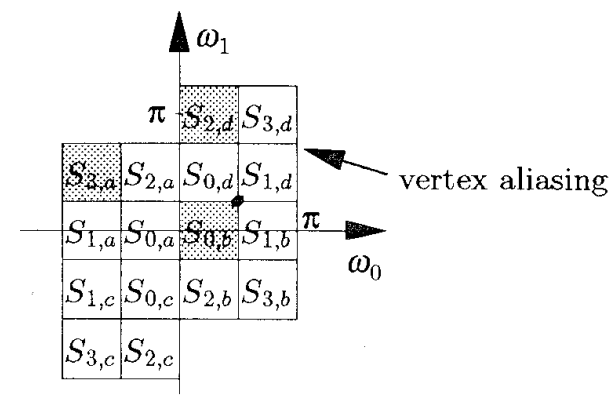

(d)

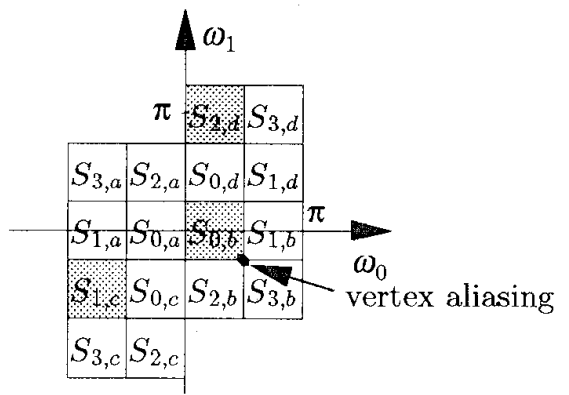

(e)

Fig. 22. Example 4.1. (a) Images of $S_{0, a}$, (b) images of $S_{2, a}$, (c) images of $S_{1, a}$, (d) images of $S_{1, c}$, and (e) images of $S_{3, a}$.

Example 3.2-Simplistic Four-Parallelogram Filter Banks: Consider an eight-channel four-parallelogram filter bank with decimation matrix

$$
\boldsymbol{M}=\underbrace{\left[\begin{array}{cc}
1 & 1 \\
-1 & 1
\end{array}\right]}_{\boldsymbol{Q}} \underbrace{\left[\begin{array}{ll}
2 & 0 \\
0 & 2
\end{array}\right]}_{\boldsymbol{\Lambda}} .
$$

We can obtain a four-parallelogram filter bank by using a tree structure filter bank with two levels (Fig. 17). For the first level of the tree, we use the diamond filter bank [9], [10]. The diamond filter bank is a two-channel system with decimation matrix $Q=\left[\begin{array}{cc}1 & 1 \\ -1 & 1\end{array}\right]$. The supports of the analysis filters in the diamond filter bank are as shown in Fig. 18(a). For the second level, we use a separable system with decimation matrix $\boldsymbol{\Lambda}=2 \boldsymbol{I}_{2}$ (Fig. 18(b)), which can be obtained as a tree structure of two 1-D two-channel filter banks. Then, the resulting analysis filters of the four-parallelogram filter banks have supports as shown Fig. 18(c). Each analysis filter $H_{m}(\boldsymbol{\omega})$ consists of four-parallelograms. We can verify that the four squares in each analysis filter are located as in (2).

Remark on Cosine-Modulated Implementation: CMFB's enjoy the advantages of low design cost and low complexity. This motivates us to consider cosine-modulated implementaion for four-parallelogram filters. In the first case, $|Q|=1$ if the two 1-D filter banks are cosine modulated, and then, the resulting 2-D nonseparable filter bank will also be cosine modulated. In the second case, $|Q|=2$, even if the two systems are cosine modulated, and the tree-structured system is not cosine modulated in general. However, in both cases, the overall systems have the desired configurations.

\section{Other Possible Four-Parallelogram Filter Banks}

In the simplistic four-parallelogram filter banks, we have constrained $L$ to be $2 I_{2}$ and the support of lowpass filter $H_{0}(\boldsymbol{\omega})$ to be a parallelogram of twice the size of the prototype parallelogram. However, for the more general fourparallelogram filter banks, the only requirement is that each filter contains four parallelograms of identical shapes. The matrix $L$ can be any integer matrix with $|L|=4$. As $L$ is an integer matrix and $|\boldsymbol{L}|=4$, there are seven possible $L A T\left(\boldsymbol{L}^{T}\right)$, as shown in Fig. 19(a)-(g). In addition, the support of the lowpass analysis filter is not necessarily a parallelogram as in simplistic four-parallelogram filter banks. Several possible supports of the first analysis filter are given in Fig. 20. In all cases of Fig. 20, the support of $H_{0}(\omega)$ consists of four connected parallelograms. In fact, any one of the four parallelograms is edge adjacent or vertex adjacent to another parallelogram. One can verify that Fig. 20 is also a complete list of connected supports for $\mathcal{A} \mathcal{F} S(\boldsymbol{M}) H_{0}(\boldsymbol{\omega})$. This follows from the fact that $L$ is an integer matrix.

Suppose we choose $L$ to be an integer matrix and choose the support of $H_{0}(\omega)$ from one of the choices in Fig. 20. In this case, it can be verified that the filter bank cannot possess both edge and vertex permissibility, except for the class of simplistic four-parallelogram filter banks in Section II and some special cases. We have not been able to analyze these special cases in a unified manner. However, exhaustive case study shows that in these special cases, the decimation matrix $\boldsymbol{M}$ is rather restricted, and the determinant of $\boldsymbol{M}$ cannot be arbitrarily large. We will look at one such example.

Example 4.1-Permissible but not Simplistic FourParallelogram Filter Bank: Consider the filter bank in Fig. 2. 
Let the decimation matrix be

$$
\boldsymbol{M}=\left[\begin{array}{cc}
1 & 1 \\
-2 & 2
\end{array}\right] \text {. }
$$

As $|M|=4$, the filter bank has four channels. If we choose $L=\left[\begin{array}{cc}2 & -1 \\ 2 & 1\end{array}\right]$, the lattice of $L^{T}$ is as shown in Fig. 19(b). In this case, the matrix $N=4 I$. We choose the configuration for the four analysis filters as in Fig. 21. Observe that the support of $H_{0}(\boldsymbol{\omega})$ is as in Fig. 20(b). The four passbands of $H_{k}(\boldsymbol{\omega})$ are labeled as $S_{k, a}, S_{k, b}, S_{k, c}, S_{k, d}$, for $k=0,1,2,3$. When decimated and expanded by $M$, each passband has three images. These images result in edge and vertex aliasing. However, we will show that these edge and vertex aliasing errors appear in pairs, and the configuration in Fig. 21 is edge and vertex permissible. Toward this end, we will verify the following. Whenever there is edge or vertex aliasing error induced by $S_{0, a}$ in a certain frequency region, we can find a similar edge or vertex aliasing error in another subband.

When the zeroth analysis filter is decimated and then expanded by $\boldsymbol{M}$, the three images of $S_{0, a}$ are at $S_{0, a}+\boldsymbol{L}^{T} \boldsymbol{k}$ for $k \in \mathcal{N}\left(\boldsymbol{M}^{T}\right)$ and $k \neq 0$ mod $\boldsymbol{M}^{T}$. One can verify that the three images are actually at $S_{2, c}, S_{1, d}$, and $S_{3, d}$ as shown in Fig. 22(a). In what follows, we discuss the aliasing errors induced by these three images:

1) Image of $S_{0, a}$ at $S_{2, c}$ : This image is edge adjacent to $S_{0, c}$ and results in edge aliasing. As $S_{0, a}$ and $S_{2, c}$ are separated by $[0-2]^{T}=L^{T}[1-1]^{T}$, this edge aliasing term contributes to aliasing transfer function $A_{(-1,1)}(\boldsymbol{\omega})$. Observe that in the second subband, passband $S_{2, a}$ has one image at $S_{0, c}$ (Fig. 22(b)), which is edge adjacent to $S_{2, c}$ and creates edge aliasing similar to that in the zeroth subband.

2) Image of $S_{0, a}$ at $S_{1, d}$ : This image is edge adjacent to $S_{0, d}$ (Fig. 22(a)) and results in edge aliasing that contributes to $A_{(1,0)}(\omega)$. In the first subband, passband $S_{1, a}$ has one image at $S_{0, d}$ (Fig. 22(c)). This image is edge adjacent to $S_{1, d}$, and a similar edge aliasing contributing to $A_{(1,0)}(\boldsymbol{\omega})$ is created. On the other hand, the image of $S_{0, a}$ at $S_{1, d}$ is also vertex adjacent to $S_{0, b}$ and results in vertex aliasing (Fig. 22(a)). However, we see that one image of $S_{1, c}$ is at $S_{0, b}$ (Fig. 22(d)) and creates aliasing similar to the vertex aliasing in the zeroth subband.

3) Image of $S_{0, a}$ at $S_{3, b}$ : This image is vertex adjacent to $S_{0, b}$ (Fig. 22(a)) and results in vertex aliasing that contributes to $A_{(0,1)}(\boldsymbol{\omega})$. We see that in the third subband, passband $S_{3, a}$ has one image at $S_{0, b}$ (Fig. 22(e)). As this image is vertex adjacent to $S_{3, b}$, it results in vertex aliasing similar to that in the zeroth subband.

In a similar manner, we can analyze the edge and vertex aliasing caused by the other passbands of $H_{0}(\boldsymbol{\omega})$ as well as the major aliasing in the other three subbands. One can show that in this example, edge and vertex aliasing always appear in pairs, and the configuration in Fig. 21 is permissible.

\section{CONCluding REMARKS}

The construction procedure of the configurations for the four-parallelogram filter banks suggests that the configurations vary with the following two factors: 1) the choice of integer matrix $\boldsymbol{L}$ and 2) the location of the four parallelograms in the support of each analysis filter. By changing these two factors, we can obtain various types of configurations. We have investigated permissibility for all those types of configurations in which the support of $H_{0}(\boldsymbol{\omega})$ is connected. In this case, the study shows that if such four-parallelogram filter banks can possess both edge permissibility and vertex permissibility, then the decimation matrix $\boldsymbol{M}$ is not arbitrary. In particular, $\boldsymbol{M}$ is either of the form $Q \Lambda$ for sone integer matrix $Q$ with $|Q|=1$ or 2, and some diagonal matrix $\Lambda$ (see (3)) or $\boldsymbol{M}$ is limited to some special cases (which are elaborated upon in Section IV). In the former event, four-parallelogram filter banks can be obtained by designing separable systems and (when $|Q|=2$ ) 2-D two-channel filter banks.

In our construction of the parallelogram prototype, we constrain $L$ to be an integer matrix. It is possible that a noninteger matrix $L$ will provide more flexibility in the design of four-parallelogram filter banks. Research in this regard is still open.

\section{REFERENCES}

[1] Y.-P. Lin and P. P. Vaidyanathan, "Theory and design of twoparallelogram filter banks," IEEE Trans. Signal Processing, this issue, pp. $2688-2706$.

[2] T. Chen and P. P. Vaidyanathan, "Consideration in multidimensional filter bank design," in Proc. Int. Symp. Circuits Syst., May 1993.

[3] M. Ikehara, "Modulated 2 dimensional perfect reconstruction FIR filter banks with permissible passbands," in Proc. Int. Conf. Acoust, Speech, Signal Processing, May 1995, pp. 1468-1471.

[4] Y.-P. Lin and P. P. Vaidyanathan, "Two-dimensional paraunitary cosine modulated perfect reconstruction filter banks," in Proc. Int. Symp. Circuits Syst., Apr. 1995, pp. 752-755.

[5] __. "New results on two-dimensional filter banks," in Proc. 29 th Ann. Asilomar Conf. Signals, Syst. Comput., Oct. 1995.

[6] J. W. Woods and S. D. O'Neil, "Subband coding of images," IEEE Trans. Accoust. Speech Signal Processing, vol. 34, pp. 1278-1288, Oct. 1986.

[7] P. P. Vaidyanathan, Multirate Systems and Filter Banks. Englewood Cliffs, NJ: Prentice-Hall, 1993.

[8] P. P. Vaidyanathan, "New results in multidimensional multirate systems," in Proc. Int. Symp. Circuits Syst., 1991

[9] M. Vetterli, "Multidimensional subband coding : some theory and algorithms," Signal Processing, vol. 6, pp. 97-112, Apr. 1984.

[10] R. Ansari and C. Guillemot, "Exact reconstruction filter banks using diamond FIR filters," in Proc. Int. Conf. New Trends Commun. Contr. Signal Processing., Turkey, July 1990.

Yuan-Pei Lin (S'93), for photograph and biography, please see p. 2705 of this issue of this Transactions.

P. P. Vaidyanathan (S'80-M'83-SM'88-F'91), for photograph and biography, please see p. 2706 of this issue of this Transactions. 